MPP-2012-154

\title{
Combined squark-squark production and decay at next-to-leading order
}

Wolfgang Hollik, Jonas M. Lindert, Davide Pagani*

Max-Planck-Institut für Physik, Föhringer Ring 6, D-80805 München, Germany

E-mail: hollik@mpp.mpg.de, lindert@mpp.mpg.de, pagani@mpp.mpg.de

In this talk we present a fully differential calculation of NLO QCD factorizable corrections to squark-squark production and direct decay into the lightest neutralino $\left(p p \rightarrow \tilde{q} \tilde{q}^{\prime} \rightarrow q \tilde{\chi}_{1}^{0} q^{\prime} \tilde{\chi}_{1}^{0}(+X)\right)$ in the narrow-width-approximation. For various benchmark scenarios, an analysis of the phenomenological impact on the experimental signature $2 j+Z_{T}(+X)$ at the LHC is presented. We compare resulting differential distributions and predictions for cutand-count searches with correspondent results in leading-order approximation rescaled by a flat K-factor.

36th International Conference on High Energy Physics,

July 4-11, 2012

Melbourne, Australia

${ }^{*}$ Speaker. 


\section{Introduction}

The hunt for a signal of new physics is amongst the main goals of the ongoing experiments at the LHC. Despite the presented impressive exclusion limits [1,2], low-scale supersymmetry (SUSY) remains one of the most appealing theories for physics beyond the Standard Model. In an R-parity conserving scenario the lightest SUSY particle (LSP) is stable and, in the case of it beeing the lightest neutralino, serves as a promising dark matter candidate. The experimental signature emerging from such a setup, presents multiple hard jets (and/or leptons) recoiling against large missing transverse momentum $\mathbb{H}_{T}$. In this talk we investigate specifically the contribution to the experimental signature $2 j(+X)+E_{T}$ from squark-squark production and direct decay into the lightest neutralino including NLO QCD corrections.

Usually, in the experimental analyses the simulation of the complete SUSY processes contributing to the given signature is performed producing events with a parton shower generator and then multiplying their weight by $\mathrm{NLO}(+\mathrm{NLL})$ QCD K-factors for just the production of squark and gluino pairs [3-6] ${ }^{1}$. Such corrections to the total cross-section are large and positive. Still, also differential corrections and corrections to the decays are generally non negligible [11].

In the calculation presented here, i.e. combined production and decay of squark pairs, differential factorizable NLO QCD corrections are included in the narrow-width-approximation (NWA). We treat independently all intermediate flavor and chirality configurations of the squarks. Such precision calculations are essential for accurate exclusion limits and potential future model determination [12].

In the following, first, we shortly discuss the theoretical framework of the calculation and second, we present numerical results. The calculation presented here is based on [13], where many more details can

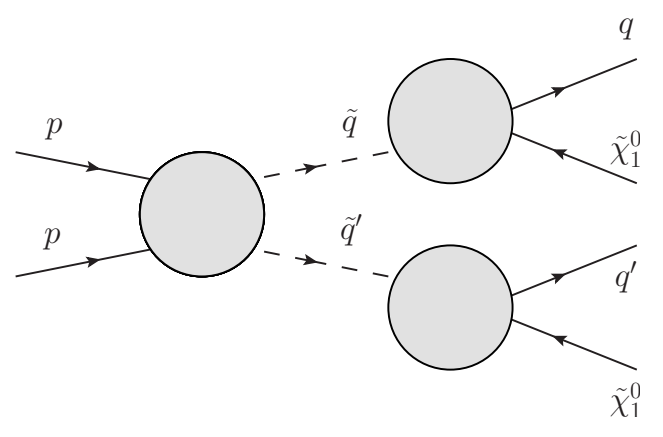

Figure 1: General structure of factorizable NLO QCD corrections to $p p \rightarrow \tilde{q} \tilde{q}^{\prime} \rightarrow$ $q \tilde{\chi}_{1}^{0} q^{\prime} \tilde{\chi}_{1}^{0}(+X)$. be found.

\section{Method}

In our calculation all squarks of the first two generations are taken into account; the cross section is given by the sum over all flavor and chirality configurations $\left(\tilde{q} \tilde{q}^{\prime}\right)$ including charge conjugated states,

$$
d \sigma=\sum_{\tilde{q} \tilde{q}^{\prime}}\left[d \sigma\left(p p \rightarrow \tilde{q} \tilde{q}^{\prime} \rightarrow q \tilde{\chi}_{1}^{0} q^{\prime} \tilde{\chi}_{1}^{0}(+X)\right)+d \sigma\left(p p \rightarrow \tilde{q}^{*} \tilde{q}^{* \prime} \rightarrow \bar{q} \tilde{\chi}_{1}^{0} \bar{q}^{\prime} \tilde{\chi}_{1}^{0}(+X)\right)\right] .
$$

The limit $\frac{\Gamma_{\tilde{q}}}{m_{\tilde{q}}} \rightarrow 0$ of the NWA, setting both squarks on-shell, allows us to write the total cross section at LO as the total cross section of just squark-squark production multiplied by the branching ratios of the two decays into the lightest neutralino. The NWA cannot in general be extended to the full set of NLO corrections, but can be applied to the subset of factorizable corrections, i.e. those

\footnotetext{
${ }^{1}$ Besides these higher order corrections in QCD also corresponding LO and NLO EW corrections have been calculated [7-10] and can become large in certain scenarios and, in general, for particular phase space regions.
} 
for production and decay as illustrated in figure 1. In a formal expansion in $\alpha_{s}$ one obtains,

$$
\begin{gathered}
d \sigma_{\mathrm{NWA}}^{(0+1)}\left(p p \rightarrow \tilde{q} \tilde{q}^{\prime} \rightarrow q \tilde{\chi}_{1}^{0} q^{\prime} \tilde{\chi}_{1}^{0}(+X)\right)=\frac{1}{\Gamma_{\tilde{q}}^{(0)} \Gamma_{\tilde{q^{\prime}}}^{(0)}}\left[d \sigma_{p p \rightarrow \tilde{q} \tilde{q}^{\prime}}^{(0)} d \Gamma_{\tilde{q} \rightarrow q \tilde{\chi}_{1}^{0}}^{(0)} d \Gamma_{\tilde{q}^{\prime} \rightarrow q^{\prime} \tilde{\chi}_{1}^{0}}^{(0)}\left(1-\frac{\Gamma_{\tilde{q}}^{(1)}}{\Gamma_{\tilde{q}}^{(0)}}-\frac{\Gamma_{\tilde{q}^{\prime}}^{(1)}}{\Gamma_{\tilde{q}^{\prime}}^{(0)}}\right)\right. \\
+d \sigma_{p p \rightarrow \tilde{q} \tilde{q}^{\prime}}^{(0)} d \Gamma_{\tilde{q} \rightarrow q \tilde{\chi}_{1}^{0}}^{(1)} d \Gamma_{\tilde{q}^{\prime} \rightarrow q^{\prime} \tilde{\chi}_{1}^{0}}^{(0)}+d \sigma_{p p \rightarrow \tilde{q} \tilde{q}^{\prime}}^{(0)} d \Gamma_{\tilde{q} \rightarrow q \tilde{\chi}_{1}^{0}}^{(0)} d \Gamma_{\tilde{q}^{\prime} \rightarrow q^{\prime} \tilde{\chi}_{1}^{0}}^{(1)} \\
\left.+d \sigma_{p p \rightarrow \tilde{q} \tilde{q}^{\prime}(X)}^{(1)} d \Gamma_{\tilde{q} \rightarrow q \tilde{\chi}_{1}^{0}}^{(0)} d \Gamma_{\tilde{q}^{\prime} \rightarrow q^{\prime} \tilde{\chi}_{1}^{0}}^{(0)}\right] .
\end{gathered}
$$

The first line corresponds to the LO differential hadronic cross-section multiplied by a global factor involving the LO total decay widths of the squarks $\Gamma_{\tilde{q}}^{(0)}$ and $\Gamma_{\tilde{q}^{\prime}}^{(0)}$ and their NLO corrections $\Gamma_{\tilde{q}}^{(1)}$ and $\Gamma_{\tilde{q}^{\prime}}^{(1)}$. The second line includes the NLO corrections to the decay distributions $d \Gamma_{\tilde{q} \rightarrow q \tilde{\chi}_{1}^{0}}^{(1)}$ and $d \Gamma_{\tilde{q}^{\prime} \rightarrow q^{\prime} \tilde{\chi}_{1}^{0}}^{(1)}$ of the two squarks, the third line contains the NLO differential corrections to the cross section $d \sigma_{p p \rightarrow \tilde{q} \tilde{q}^{\prime}(X)}^{(1)}$. We compute all these ingredients at fully differential level and combine them numerically according to eq. (2.2), obtaining in this way the full set of factorizable NLO corrections to squark-squark production and decay ${ }^{2}$.

\section{Phenomenological evaluation}

We present numerical results for three different benchmark scenarios: the CMSSM points SPS1a, 10.1.5, and a rather compressed scenario p19MSSM1A. Although the point SPS1a has already been excluded by recent searches, in the literature many results have been presented for this benchmark scenario. The other two points have not been explicitly excluded; they can, however, be tested with current available data from the $8 \mathrm{TeV}$ run of the LHC. Masses of the particles entering the calculation are listed in Tab. 1.

\begin{tabular}{c||c|c|c|c|c|c}
\hline benchmarkpoint & $\tilde{u}_{L}$ & $\tilde{u}_{R}$ & $\tilde{d}_{L}$ & $\tilde{d}_{R}$ & $\tilde{g}$ & $\tilde{\chi}_{1}^{0}$ \\
\hline SPS1a & 563.6 & 546.7 & 569.0 & 546.6 & 608.5 & 97.0 \\
\hline 10.1 .5 & 1437.7 & 1382.3 & 1439.7 & 1376.9 & 1568.6 & 291.3 \\
\hline p19MSSM1A & 339.6 & 394.8 & 348.3 & 392.7 & 414.7 & 299.1 \\
\hline
\end{tabular}

Table 1: On-shell masses of squarks, gluinos, and the lightest neutralino within the different SUSY scenarios considered. All masses are given in $\mathrm{GeV}$.

We cluster partons into jets using the anti- $\mathrm{k}_{T}$ algorithm and apply preselection cuts as used by ATLAS and CMS (details can be found in [13]).

\subsection{Differential distributions}

In figure 2 we show band plots at $14 \mathrm{TeV}$ for the differential cross-sections in $p_{T}$ of the hardest jet and in $E_{T}$. The bands are obtained varying the common renormalization and factorization scale between $\mu / 2$ and $2 \mu$, with $\mu$ equal to the average mass of the squarks. Blue bands represent the

\footnotetext{
${ }^{2}$ Non-factorizable corrections like interactions between initial and final state quarks, formally at the same order of perturbation theory, are of less importance.
} 

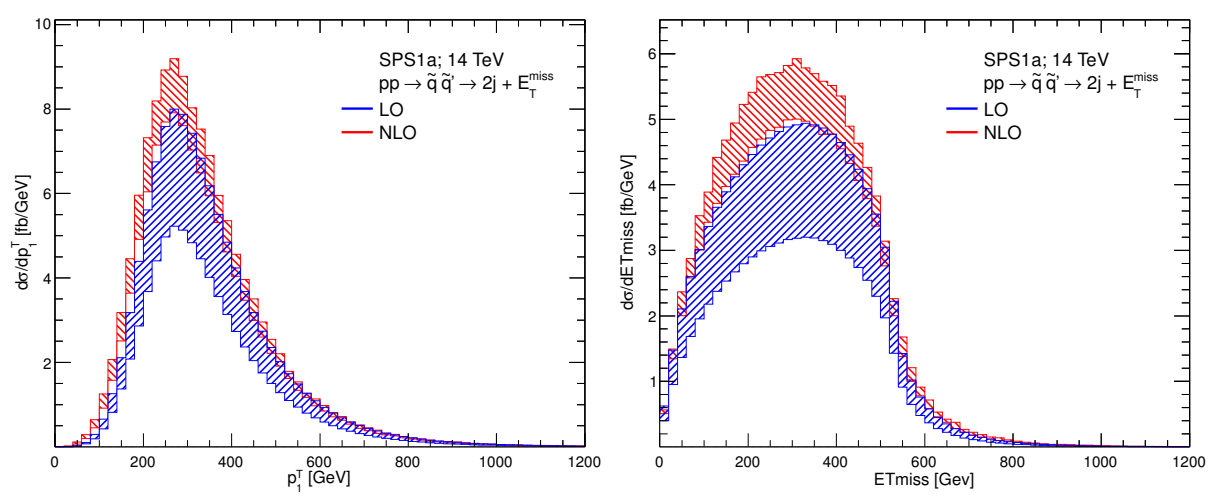

Figure 2: Differential distributions in $p_{1}^{\mathrm{T}}, \not_{T}$ (in fb/GeV) for SPS1a at $\sqrt{S}=14 \mathrm{TeV}$ : LO (blue) and NLO (red). The common renormalization and factorization scale $\mu$ is varied between $\overline{m_{\tilde{q}}} / 2$ and $2 \overline{m_{\tilde{q}}}$.
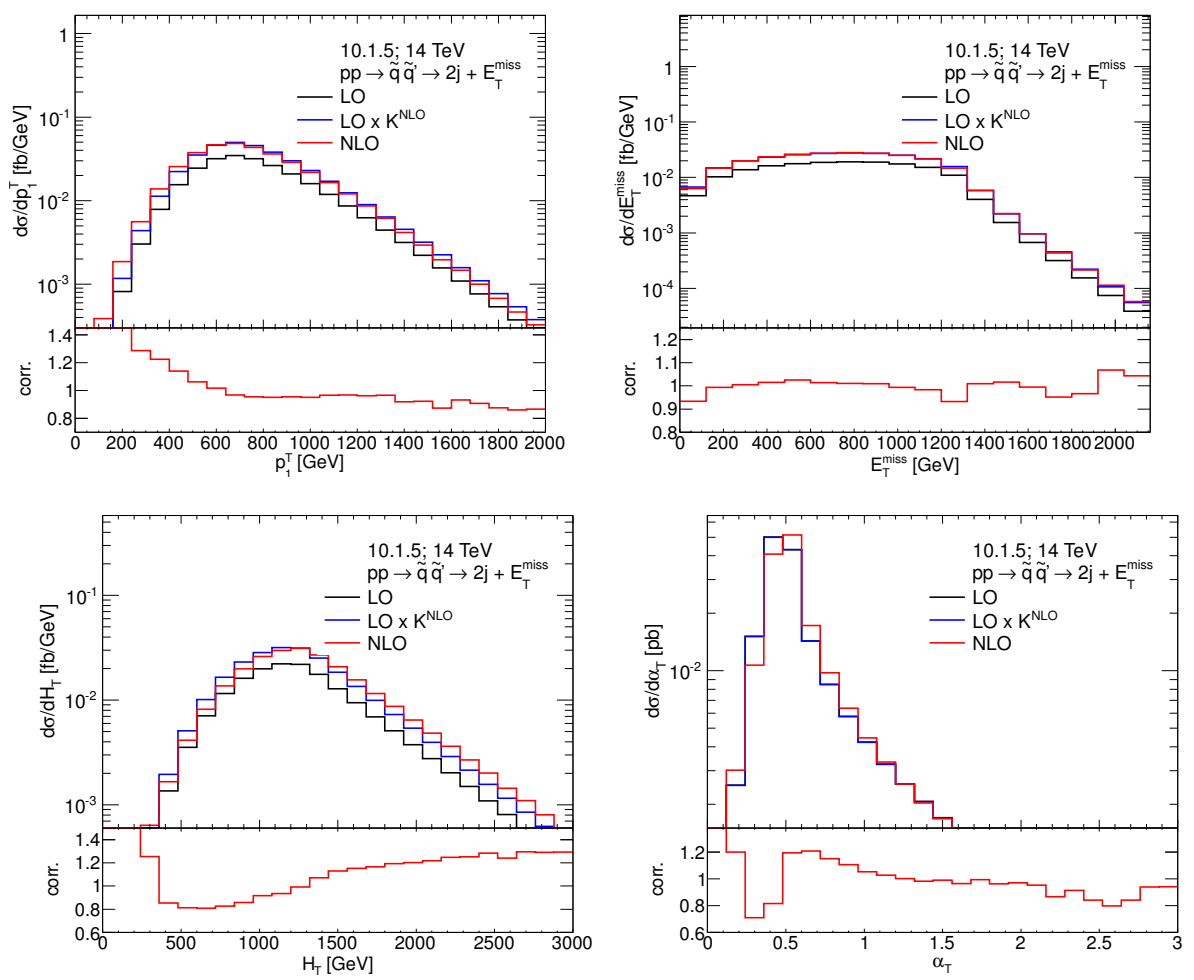

Figure 3: Differential distributions for the benchmark point 10.1 .5 at a center of mass energy $\sqrt{S}=14$. Upper part: LO (black), NLO (red) and LO rescaled by the ratio $K^{\mathrm{NLO}}$ (blue). Lower part: $\frac{\mathrm{NLO}}{\mathrm{LO} \cdot K^{\mathrm{NLO}}}$.

LO results, red bands include the NLO factorizable corrections. As expected, the dependence on the variation of the scale is reduced including the NLO corrections. In this way a theoretical error can be estimated.

In figure 3 we illustrate the impact of NLO factorizable corrections on the shape of distributions. Differential cross sections for CMSSM 10.1.5 at $14 \mathrm{TeV}$ in $p_{1}^{T}, \not_{T}, H_{T}$ and $\alpha_{T}$ are presented in three different approximations: LO (black), NLO (red), and LO rescaled by the ratio $K^{\mathrm{NLO}}$ be- 

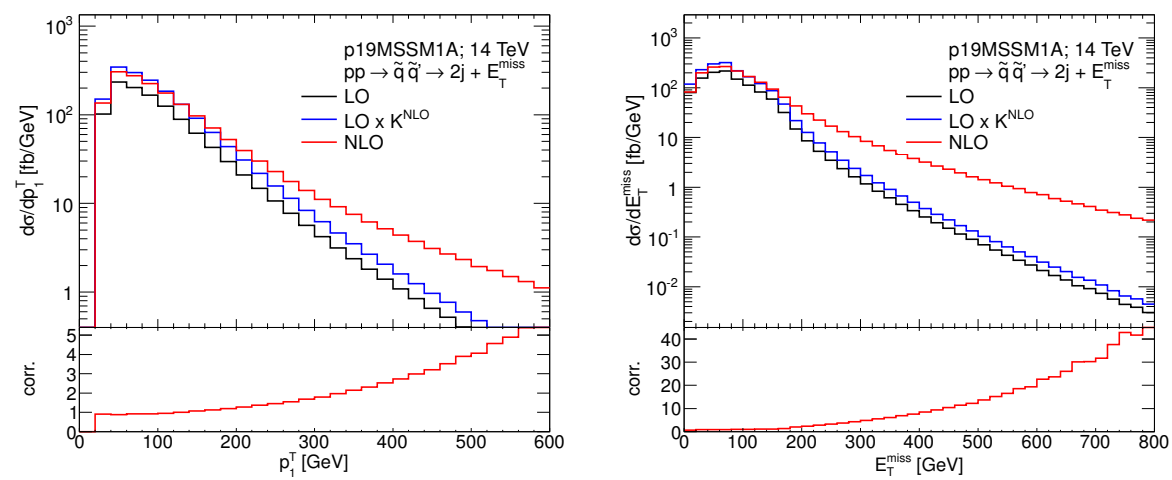

Figure 4: Differential distributions in $p_{1}^{\mathrm{T}}$ and in $Z_{T}$ for the benchmark point p19MSSM1A at a center of mass energy $\sqrt{S}=14$. Upper part: LO (black), NLO (red) and LO rescaled by the ratio $K^{\mathrm{NLO}}$ (blue). Lower part: $\frac{\mathrm{NLO}}{\mathrm{LO} \cdot K^{\mathrm{NLO}}}$.

tween the integrated NLO and LO cross section (blue). In the lower part of the plots, the ratio between the NLO and the rescaled $\mathrm{LO} \times K^{\mathrm{NLO}}$ is shown. In this way differences exclusively in the shapes are highlighted. The observables $H_{T}$ and $\alpha_{T}$ are used by CMS in their searches for SUSY signatures. The signal region in cut-and-count searches is defined as $\alpha_{T}<0.55$. Corrections in shapes change sign around this value.

The same kind of plots for the benchmark point p19MSSM1A at $14 \mathrm{TeV}$, differential in $p_{1}^{T}$ and $Z_{T}$, are shown in figure 4 . For such a compressed spectrum the mass gap between the squarks and the lightest neutralino is much smaller than in the previous two cases. Jets from the decays are soft, namely of the order of the applied jet cuts, so their detection is extremely challenging. Still, even in such situations the experiments might be sensitive [14]. In figure 4 again distributions in $p_{1}^{T} \mathbb{E}_{T}$ are shown. Corrections purely in shapes grow drastically in the tail of the distributions. These plots indicate that a LO approximation is insufficient for a realistic description of the hard jets. Such kind of behavior was already discussed in [15]; here we present a more complete analysis at NLO QCD.

\subsection{Cut-and-count searches}

Within the LHC collaborations, supersymmetric particles are searched for as an excesses of events in signal regions designed to reduce the relative background contribution from Standard Model processes. ATLAS and CMS have performed such cut-and-count searches for the experimental signature 2 jets $+Z_{T}$ in different signal regions. In [13] we investigate the impact of factorizable NLO QCD corrections to these signal regions from squark-squark production and decay in detail. In this way one can estimate the error introduced by using a rescaled LO result compared to the fully differential NLO calculation as presented here. For the parameter points SPS1a and 10.1.5 and applying cuts as done by the ATLAS collaboration this error is at most of the order of $10 \%$. For the cuts used by CMS this error can be as large as 30\%. As already shown in figure 4, a LO prediction is completely unreliable for a compressed spectrum like p19MSSM1A. This is reflected in corrections of $\mathscr{O}\left(10^{2}-10^{4}\right)$ for the investigated signal regions. 


\section{Conclusions}

In this talk we presented results from a new calculation that combines QCD corrections in production and decay of squark pairs at the LHC. Implications for differential distributions for the experimental signature $2 j+Z_{T}(+X)$ and for searches for supersymmetry at the LHC have been investigated. In general, NLO corrections are not negligible for setting precise limits on the sparticle masses and model parameters. For the same purpose, a fully differential calculation of NLO QCD corrections for production and decay of all squark and gluino channels is desirable.

\section{References}

[1] G. Aad et al. Search for squarks and gluinos using final states with jets and missing transverse momentum with the ATLAS detector in $\mathrm{sqrt}(\mathrm{s})=7 \mathrm{TeV}$ proton-proton collisions. Phys.Lett., B710:67-85, 2012.

[2] V. Khachatryan et al. Search for Supersymmetry in pp Collisions at $7 \mathrm{TeV}$ in Events with Jets and Missing Transverse Energy. Phys.Lett., B698:196-218, 2011.

[3] W. Beenakker, R. Hopker, M. Spira, and P. M. Zerwas. Squark and gluino production at hadron colliders. Nucl. Phys., B492:51-103, 1997.

[4] W. Beenakker et al. Soft-gluon resummation for squark and gluino hadroproduction. JHEP, 12:041, 2009.

[5] M. Beneke, P. Falgari, and C. Schwinn. Threshold resummation for pair production of coloured heavy (s)particles at hadron colliders. Nucl. Phys., B842:414-474, 2011.

[6] P. Falgari, C. Schwinn, and C. Wever. NLL soft and Coulomb resummation for squark and gluino production at the LHC. JHEP, 06:052, 2012.

[7] S. Bornhauser, M. Drees, H. K. Dreiner, and J. S. Kim. Electroweak Contributions to Squark Pair Production at the LHC. Phys. Rev., D76:095020, 2007.

[8] W. Hollik and E. Mirabella. Squark anti-squark pair production at the LHC: the electroweak contribution. JHEP, 12:087, 2008.

[9] W. Hollik, E. Mirabella, and M. K. Trenkel. Electroweak contributions to squark-gluino production at the LHC. JHEP, 02:002, 2009.

[10] J. Germer, W. Hollik, E. Mirabella, and M. K. Trenkel. Hadronic production of squark-squark pairs: The electroweak contributions. JHEP, 08:023, 2010.

[11] A. Djouadi, W. Hollik, and C. Junger. QCD corrections to scalar quark decays. Phys. Rev., D55:6975-6985, 1997.

[12] H. K. Dreiner, M. Kramer, J. M. Lindert, and B. O'Leary. SUSY parameter determination at the LHC using cross sections and kinematic edges. JHEP, 04:109, 2010.

[13] W. Hollik, J. M. Lindert, and D. Pagani. NLO corrections to squark-squark production and decay at the LHC. JHEP, 03:139, 2013.

[14] H. K. Dreiner, M. Kramer, and J. Tattersall. How low can SUSY go? Matching, monojets and compressed spectra. Europhys.Lett., 99:61001, 2012.

[15] J. Alwall, S. de Visscher, and F. Maltoni. QCD radiation in the production of heavy colored particles at the LHC. JHEP, 02:017, 2009. 